\title{
Impact of the Antenna-Body Distance on the WBAN Channel Characteristics
}

\author{
Mariella Särestöniemi \\ Centre for Wireless Comminucations \\ Faculty of Information Technology and \\ Electrical Engineering \\ Univeristy of Oulu \\ Oulu, Finland \\ mariella.sarestoniemi@oulu.fi \\ Matti Hämäläinen \\ Centre for Wireless Comminucations \\ Faculty of Information Technology and \\ Electrical Engineering \\ Univeristy of Oulu \\ Oulu, Finland \\ matti.hamalainen@oulu.fi
}

\author{
Chaïmaâ Kissi \\ Electronics and Telecommunication \\ Systems Research Group, National \\ School of Applied Sciences (ENSA) \\ Ibn Tofail University \\ Kenitra, Morocco \\ chaimaakissi1@gmail.com \\ Jari Iinatti \\ Centre for Wireless Comminucations \\ Faculty of Information Technology and \\ Electrical Engineering \\ Univeristy of Oulu \\ Oulu, Finland \\ jari.iinatti@oulu.fi
}

\author{
Carlos Pomalaza Raez \\ Department of Electrical and Computer \\ Engineering \\ Purdue University \\ Fort Wayne, Indiana 46805, USA \\ cpomalaz@purdue.edu
}

\begin{abstract}
This paper presents a simulation based study on the impact of the antenna-body distance on the ultra wideband (uwb) on-body radio channel characteristics. The antenna used in this study is a recently published highly directional cavitybacked uwb antenna, designed for inbody communications, e.g. capsule endoscope localization. The on-body channel characteristics are evaluated both in frequency and time domains with several antenna-body distances as well as with several antenna location options. Furthermore, the impact of the antenna-body distance is evaluated by studying 3D power patterns, which also provide information about the propagation depth within tissues in different antenna-body distances. It is shown that antenna-body distance has a significant impact on the on-body channel characteristics. In time domain the difference can be $15 \mathrm{~dB}$ between the main peaks of the impulse responses whereas in frequency domain, the path loss difference can be even $30 \mathrm{~dB}$ within the frequency band of interest. Besides, antenna-body distance has a clear impact on the propagation depth inside the tissues as well.
\end{abstract}

Keywords-capsule endoscopy, power flow, propagation depth, radio channel, ultra wideband, wireless body area networks

\section{INTRODUCTION}

Recently there has been significant interest on the health monitoring devices and applications, calling for further investigations on the antenna and channel characteristics in wireless body area networks (WBAN) [1]-[7]. Antenna behavior differs in the close vicinity of human body from its normal behavior [8]. Usually the antenna properties improve when the antenna is placed further from the body. However, for several practical WBAN scenarios it is essential to have as small antenna-to-body distance as possible. Especially, the antennas in sensor nodes should work well in the close vicinity of the human body as several sensor nodes may be deployed on the body. On other hand, the antenna on a monitoring device could have a slightly larger antenna-tobody distance in practice.

There are several studies on the impact of antenna-body distance on the antenna matching, e.g. in [7], [9], [10-12]. However, up to our knowledge there is just a few studies presenting results on impact of the antenna-body distance on the channel characteristics. Among the first pioneering work in this area is [8], which presents study on the effects of the human body on UWB off-body propagation in an indoor environment. In [4], we presented channel characteristics to certain antenna location options with antenna-body distance $30 \mathrm{~mm}$, in which the antenna is known to work optimally [6] and compared the results to a realistic scenario with antennabody distance $4 \mathrm{~mm}$.

The main contribution of this paper is to present a comprehensive simulation based study on the impact of antenna-body distance on the channel characteristics: propagation between the on-body transmitter and receiver antennas as well as propagation depth inside the tissues with different antenna-body distances. The simulations are conducted using a multilayer model of the human abdomen area as well as a voxel model having all the relevant tissues for this study case.

This paper is organized as follows: Section II presents the study case describing the simulation models, antennas, and antenna locations used in the simulations. Section III presents the layer-model simulation results for different antenna-body distances with different antenna location scenarios. Simulation results with a voxel model are presented in Section IV. Conclusions and future works are discussed in Section V.

\section{STUDY CASE}

\section{A. Simulation models}

This study has been conducted using electromagnetic propagation simulation software CST [13], which is based on finite integration technique (FIT). Simulations are carried out mainly using a planar multilayer model of the human abdomen area, which is presented in Figure 1. Besides of transmitter and receiver antennas (Tx, Rx), the model consists of tissue layers: skin, subcutaneous fat, muscle, visceral fat, small intestine wall and content layers, for which the dielectric properties has been obtained from [14]. The dielectric properties and thicknesses of the layers are presented in Table I. Additionally, we use a female voxel model, Laura, which is obtained from CST's voxel family library [13], to evaluate the impact of the antenna-body distance with a more realistic simulation model. 


\section{B. Antenna}

In this study, we used a cavity-backed antenna [6] designed for inbody communications at the low band of UWB band 3.75-4.25 GHz. Figure 2 presents the simulation model of the antenna. For this antenna, we have modeled the coaxial cable to the antenna feeding to get better correspondence between the simulation and measurement results presented e.g. in [5], [7].

In this study case, the antenna-body distance $d$ corresponds to the distance between the skin and the antenna cable, as shown in Figure $2 \mathrm{a}$ and $\mathrm{b}$ for the layer model and the voxel model, respectively. The distance between the antenna radiator and the outer part of the cable is $d_{\mathrm{r}-\mathrm{c}}=3.6 \mathrm{~mm}$. Hence, the distance between antenna radiator and skin is $d_{\mathrm{r}-\mathrm{s}}=d_{\mathrm{r}-\mathrm{c}}+d$. In the notations of this paper, we selected to use the distance $d$ instead $d_{\mathrm{r}-\mathrm{s}}$ to emphasize which is minimum possible distance between the antenna model and skin.

a)

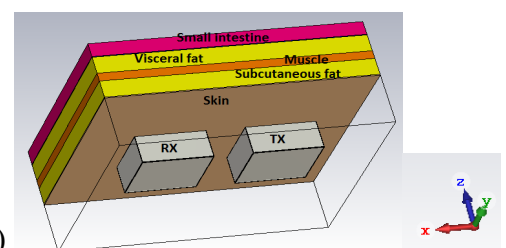

b)

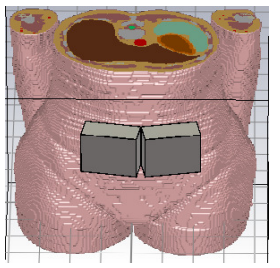

Figure 1. a) Multilayer model of the human abdomen area, b). torso of a female voxel model

TABLE I: THICKNESSES OF LAYERS AND DIELECTRIC PROPERTIES AT $4 \mathrm{GHz}$

\begin{tabular}{|l|l|l|l|}
\hline Tissue & $\begin{array}{l}\text { Thickness } \\
(\mathrm{mm})\end{array}$ & $\begin{array}{l}\text { Permit- } \\
\text { tivity }\end{array}$ & $\begin{array}{l}\text { Conductivity } \\
(\mathrm{S} / \mathrm{m})\end{array}$ \\
\hline Skin & 1.4 & 40.85 & 2.701 \\
\hline Subcutaneous fat & 20 & 5.125 & 0.1829 \\
\hline Muscle & 12 & 50.82 & 3.015 \\
\hline Visceral fat & 20 & 5.125 & 0.1829 \\
\hline SI wall & 1 & 50.82 & 3.015 \\
\hline SI content & 20 & 51.63 & 4.622 \\
\hline
\end{tabular}

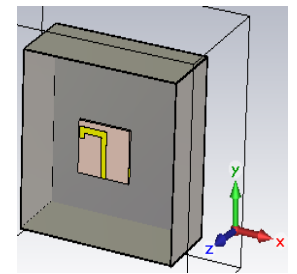

Figure 2. The cavity-backed on-body antenna.

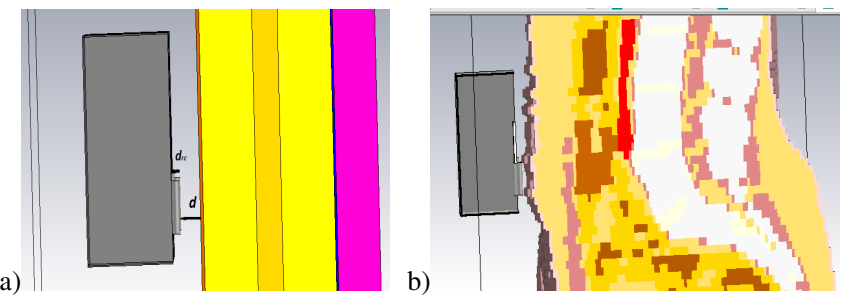

Figure 3.Antenna located on a) layer model surface, and b) voxel model surface.

\section{Antenna location options}

The impact of antenna-body distance on the channel characteristics is evaluated with four different antenna location options. Antenna location option 1 is side-by-side case, in which antenna separation distance $d_{\mathrm{a}}=0 \mathrm{~cm}$. The distance $d_{\mathrm{a}}$ is measured from the edges of the cavities. In this case, the distance between the antennas' feeding points $d_{\mathrm{f}}=3.96 \mathrm{~cm}$.
In the antenna location option 2, the antennas are horizontally one the same line having $d_{\mathrm{a}}=2 \mathrm{~cm}$ and $d_{\mathrm{f}}=11.13$ $\mathrm{cm}$. In the antenna location options 3 and 4, the distance between the antennas' cavity edges and feeding points are $d_{\mathrm{a}}=5 \mathrm{~cm}, d_{\mathrm{f}}=14.13 \mathrm{~cm}$ and $d_{\mathrm{a}}=8 \mathrm{~cm}, d_{\mathrm{f}}=17.13 \mathrm{~cm}$, respectively.

\section{SIMULATION RESULTS WITH LAYER MODEL}

In this section, we evaluate the impact of the antenna-body distance by studying frequency and time domain channel characteristics, as well as 3D-power patterns in the selected antenna-body distances $d=0 \mathrm{~mm}, \quad d=2 \mathrm{~mm}, \quad d=4 \mathrm{~mm}$, $d=10 \mathrm{~mm}$, and $d=30 \mathrm{~mm}$.

\section{A. Side by side, antenna separation distance $0 \mathrm{~cm}$}

The antenna reflection coefficients $\mathrm{S} 11$, frequency domain channel parameter S21 (path loss), and time domain channel characteristics for different antenna-body distances are presented in Figure 4a-c, respectively. Notation S2,1 means channel parameter $\mathrm{S} 21$, i.e., the channel frequency response as the antenna model-skin distance $d=0 \mathrm{~mm}$. The rest of the distances are marked separately in the figure as underscores. The S11 for antenna-body distances $4 \mathrm{~mm}$ and $30 \mathrm{~mm}$ are studied in detail in [6], this paper just briefly presents the S11s to explain the difference the S21 behavior. As expected from the results presented in [6], S11 improves as the antenna-body distance increases. The most dramatic difference is between $d=0 \mathrm{~mm}$ and $d=2 \mathrm{~mm}$. In general, S11 changes a lot with the antenna-body distance.

As we study the S21-parameters for different antennabody distances, we can see clear variation in the path losses. Interestingly, the path loss is lower for the case of $d=10 \mathrm{~mm}$ than for the case of $d=30 \mathrm{~mm}$, whereas in many cases the path loss is considered to be larger as the antenna-body distance is smaller [9]. According to the time domain results presented in Figure $4 \mathrm{~b}$, the channel of the case of $d=10 \mathrm{~mm}$ is strong: between time period $1.5-3 \mathrm{~ns}$, the main peak is clearly higher than that of the other cases, even the case of $d=30 \mathrm{~mm}$. The difference between the main peaks of the weakest and strongest channels is roughly $10 \mathrm{~dB}$.

The 3D-power pattern presentation, presented for this case at $f=4 \mathrm{Ghz}$ within $\mathrm{dB}$ range $0--40 \mathrm{~dB}$ in Figure 5, describes well how the power is spread from the Tx-antenna into different directions with different antenna-body distances. In the figures, the antennas can be seen as light boxes inside the patterns, Tx on the right side, Rx on the left side. Below the antennas is the layer model, in which the lowest layer corresponds to small intestine layer, as presented in Figure 1. Power patterns give explanation why the channel strength varies from case to case. The strength of the power is depicted with colors, in this case red and orange illustrate strongest power flow. However, in this antenna location option case, the power patterns at $f=4 \mathrm{GHz}$ do not explain reason why the channel of $d=10 \mathrm{~mm}$ case is clearly stronger that of the $d=30 \mathrm{~mm}$ case, since the power patterns between $\mathrm{Tx}$ and $\mathrm{Rx}$ antennas are quite similar. Presumably, the 3D-power patterns from the other frequencies, larger $\mathrm{dB}$ range, or directivity or realized gain presented in [7] could explain the reason of the difference, but due to lack of the space, they are not studied separately in this paper.

With 3D power patterns, we can easily observe the propagation depth inside the tissues within the selected $\mathrm{dB}$ 
range. In this antenna location option's case, the small intestine layer is achieved at least partly with all the antenna-body distance cases within the selected $\mathrm{dB}$ range 0 $-40 \mathrm{~dB}$. However, coverage in the small intestine layer is narrow, since it covers mainly the area below the Tx and only slightly below the Rx. However, if we set the $\mathrm{dB}$ range cover until $-50 \mathrm{~dB}$, we get better coverage in all the cases. Furthermore, the frequency has a clear impact on the coverage. The impact of frequency and $\mathrm{dB}$ range will be discussed more in detail with examples, at the end of this section. In the following, we focus on the power patterns at $f=4 \mathrm{GHz}$, which is antenna's operational frequency.

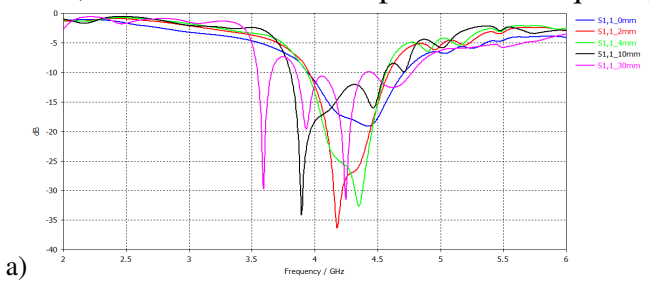

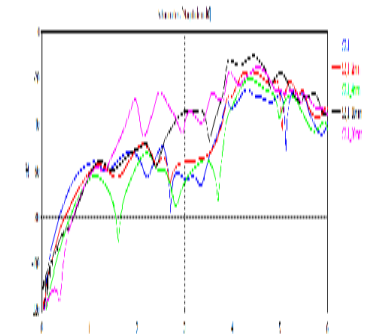

b)

Figure 4. Channel characteristics with different antenna-body distances in a) frequency and b) time domain as the antenna separation distance is $0 \mathrm{~cm}$.
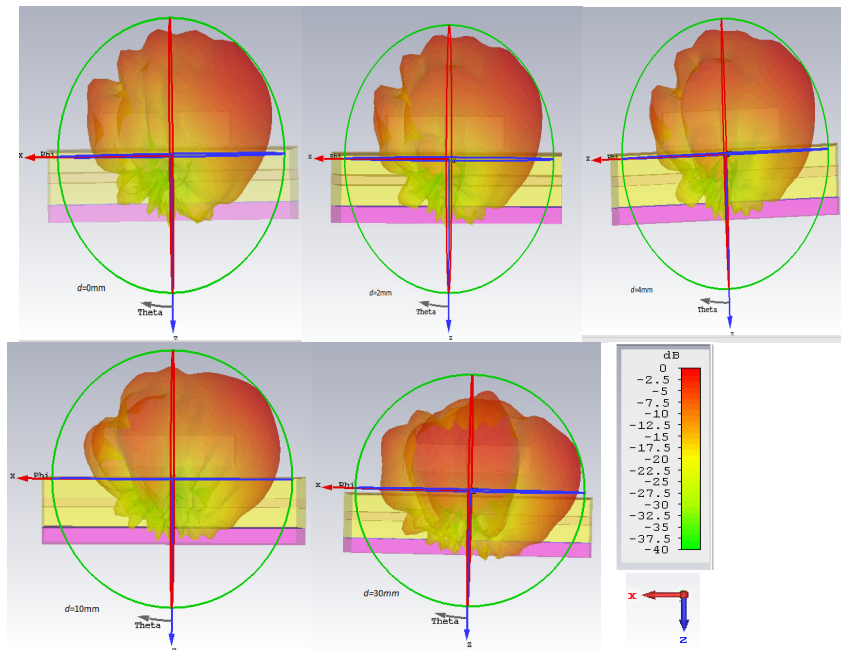

Figure 5. Power pattern at $f=4 \mathrm{GHz}$ for different antenna-body distances $d$ as the antenna separation distance is $0 \mathrm{~cm}$.

\section{B. Antenna separation distance $2 \mathrm{~cm}$}

Next, we evaluate the impact of the antenna-body distance in the antenna location option 2, i.e., as the antenna separation distance is $2 \mathrm{~cm}$. The frequency and time domain results are presented in Figure $6 \mathrm{a}$ and $6 \mathrm{~b}$, respectively. In this case, the variation between the antenna-body distance cases is even larger than in the case of antenna location option 1. In frequency domain within the frequency band of interest, the variation between the strongest and weakest channel is around $30 \mathrm{~dB}$, whereas in time domain, the difference between the main peaks of the strongest and the weakest impulse responses is around $15 \mathrm{~dB}$. Interestingly, also in this antenna location options case, the strongest channel is obtained as the antenna-body location distance is $10 \mathrm{~mm}$. When studying the power patterns presented in Figure 7, we can see that the tendency between different antenna skin distances are similar to the case of antenna location option 1, though the reachability of small intestine layer is weaker compared to the first antenna location option. In the case of $d=10 \mathrm{~mm}$, only the upper part of the small intestine layer is achieved within the $\mathrm{dB}$ range $0--40$ $\mathrm{dB}$. Similar to the antenna location option 1 , the best coverage is obtained with $d=30 \mathrm{~mm}$.

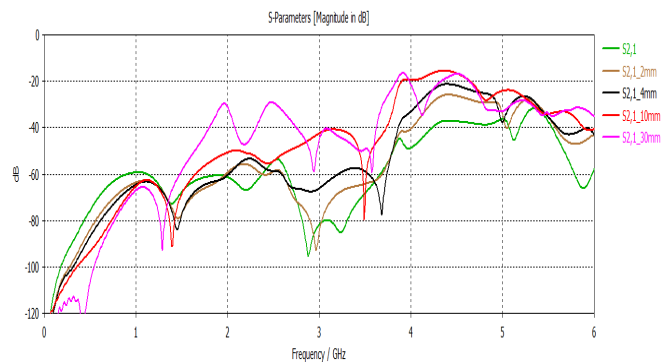

a)

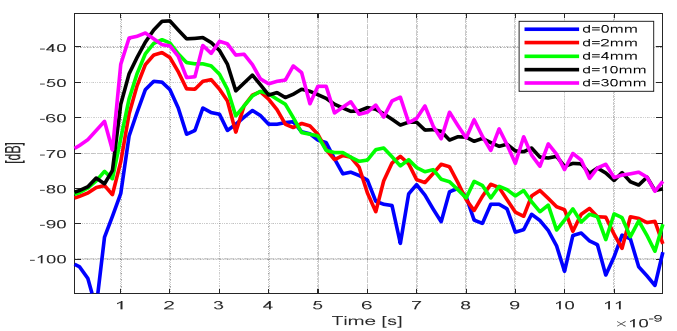

b)

Figure 6. Channel characteristics with different antenna-body distances in a) frequency and b) time domain as the antenna separation distance is $2 \mathrm{~cm}$.

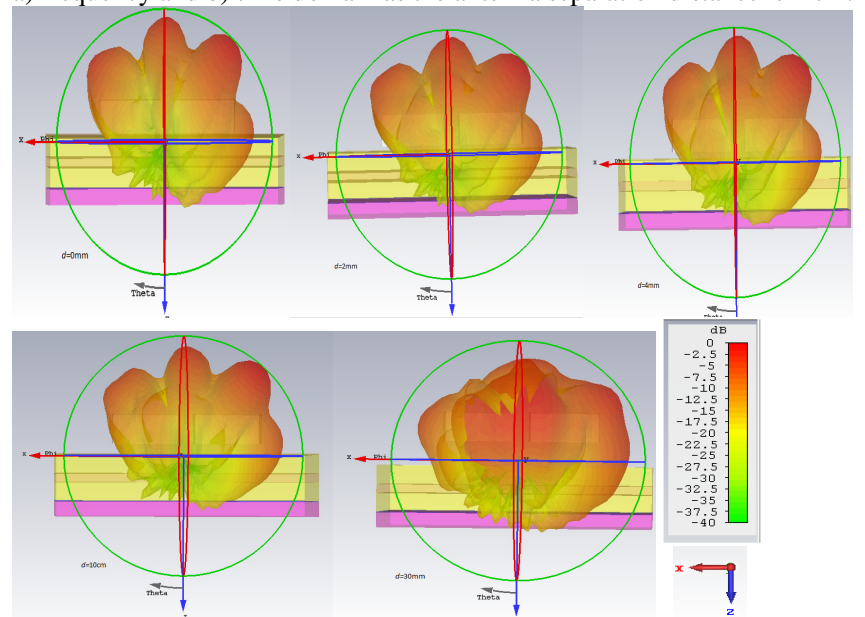

Figure 7. Power patterns at $f=4 \mathrm{GHz}$ for different antenna-body distances as the antenna separation distance is $2 \mathrm{~cm}$.

\section{Antenna separation distance $5 \mathrm{~cm}$}

Next, the channel characteristics are evaluated as the antennas separation distance is $5 \mathrm{~cm}$. The frequency and time domain results are presented in Figure $8 \mathrm{a}$ and $8 \mathrm{~b}$, respectively. Also in this case we can see clearly impact of the antenna-body distance both in frequency and time domain. In the frequency domain, within the frequency bandwidth of interest, we can see over $20 \mathrm{~dB}$ variation between different antenna-body separation cases. The case $d=30 \mathrm{~mm}$ is clearly at the highest level. However, at 
$f=4.167 \mathrm{GHz}$, which belongs to the frequency band of interest, there is a clear notch for the case of $d=30 \mathrm{~mm}$. These kinds of notches are explained with the radiation patterns as shown in [7].

When studying the impulse responses in time domain, we can see also in this case that level of the main peak increases as the distance between the antenna and skin increases. However, the channel with antenna-body distance $d=30 \mathrm{~mm}$ is at the higher level than with the case of $d=10 \mathrm{~mm}$. Only at the time instant $t=0.22 \mathrm{~ns}$, the peak of case $d=10 \mathrm{~mm}$ is slightly higher to that of $d=30 \mathrm{~mm}$ case. Besides, the side peaks of the case of $d=30 \mathrm{~mm}$ are at significantly higher level than in the other cases.
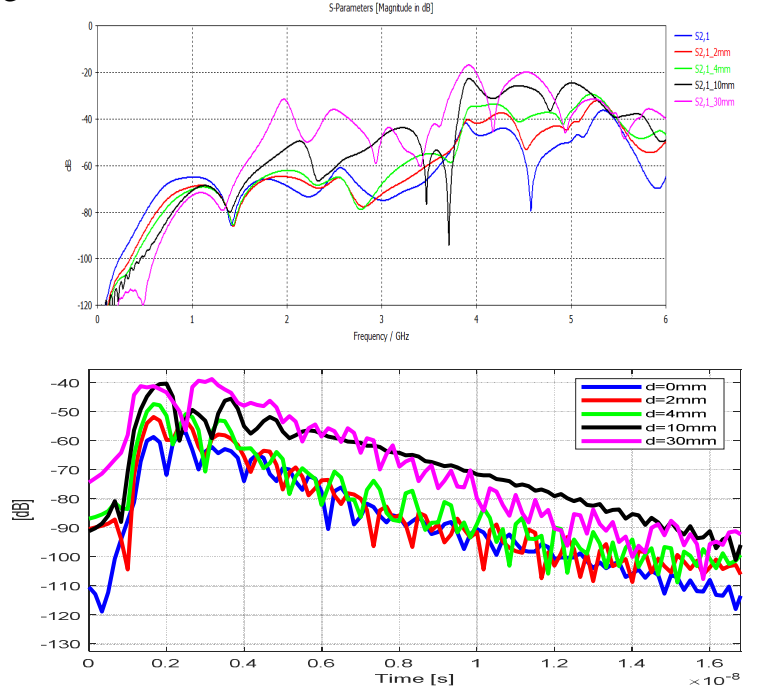

Figure 8. Channel characteristics with different antenna-body distances in a) frequency and b) time domain as the antenna separation distance is $5 \mathrm{~cm}$.

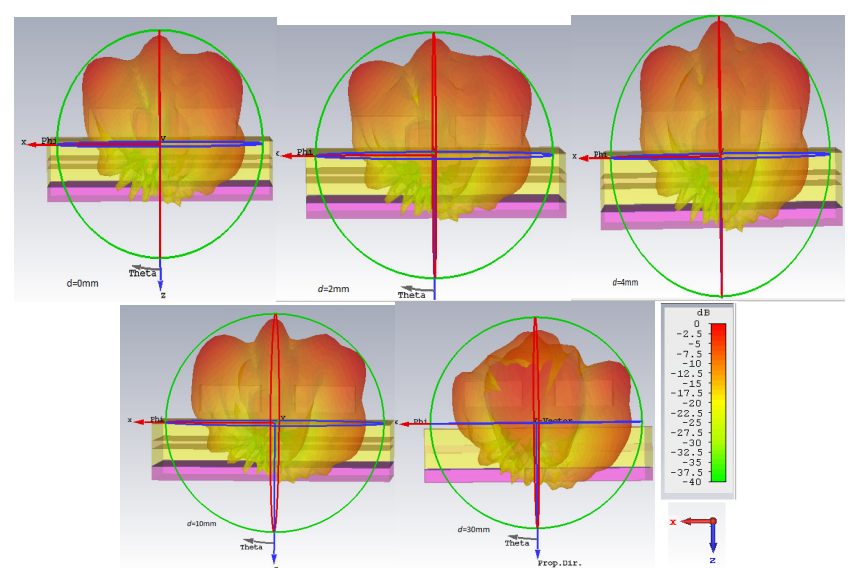

Figure 9. Power pattern for different antenna-body distances as the antenna separation distance is $5 \mathrm{~cm}$.

From the 3D-power pattern figures presented in Figure 9, we can see some differences in terms of power flow between the $\mathrm{Tx}$ and $\mathrm{Rx}$ antennas. However, the case of $d=30 \mathrm{~mm}$ is the most different from the others: there we can see clearly strong power flow from $\mathrm{Tx}$ to $\mathrm{Rx}$ since the power pattern is mostly as red or orange. This explains the reason for frequency and time domain channel characteristics of the case on $d=30 \mathrm{~mm}$ being much higher level than those of the other cases.

Furthermore, it is noted that the small intestine tissue layer is achieved well in all the antenna-body distance cases within the selected $\mathrm{dB}$ range $0--40 \mathrm{~dB}$. In the case of $d=30 \mathrm{~mm}$, the small intestine layer is covered at widest and actually the power limit $-40 \mathrm{~dB}$ is reached even below the small intestine layer. Interestingly, the coverage of the small intestine layer is weakest in the case of the antenna-body distance $d=10 \mathrm{~mm}$.

\section{Antenna separation distance $8 \mathrm{~cm}$}

Finally, we study the channel characteristics in the case of antenna separation distance $8 \mathrm{~cm}$. The frequency and time domain results are presented in Figure 10a and b, respectively. Similarly to the previous antenna location option cases, we can see clearly impact of the antenna-body distance both in frequency and time domain. In the frequency domain, within the frequency bandwidth of interest, we can see even $30 \mathrm{~dB}$ variation between different values of $d$. As contrast for the previous antenna location options, now the $\mathrm{S} 21$ of the case $d=30 \mathrm{~mm}$ is clearly at the highest level, except in the same notch frequency at 4.16 $\mathrm{GHz}$ as in the antenna location option 3. The impact of the antenna-body distance can clearly be seen also in time domain results presented in Figure 10b. Interestingly, the level of the main peaks of the cases $d=10 \mathrm{~mm}$ and $d=30 \mathrm{~mm}$ is on the same level, but there is a clear difference between the level of the side peaks. For the case $d=30 \mathrm{~mm}$ the side peaks are at remarkably higher level.

Within the selected $\mathrm{dB}$ range, power flow patterns are surprisingly similar for the cases $d=0 \mathrm{~mm}, d=2 \mathrm{~mm}$, and $d=4 \mathrm{~mm}$ in terms of power flow between the Tx and Rx antennas as well as of propagation depth. In the case of $d=10 \mathrm{~mm}$, propagation depth is slightly weaker than with the smaller antenna-body distances, whereas the power flow between $\mathrm{Tx}$ and $\mathrm{Rx}$ antennas is stronger. Instead, propagation pattern for the case of $d=30 \mathrm{~mm}$ is clearly different from the other cases: power flow between $\mathrm{Tx}$ and $\mathrm{Rx}$ antennas is very strong as well as small intestine coverage is clearly wider.

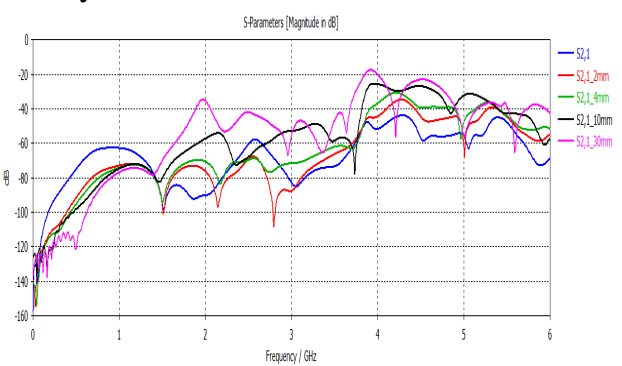

a)

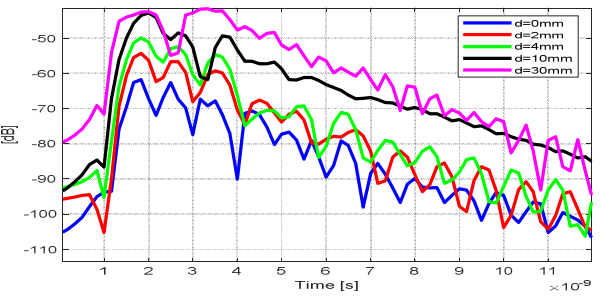

b)

Figure 10. Channel characteristics with different antenna-body distances in a) frequency and b) time domain as the antenna separation distance is $8 \mathrm{~cm}$.

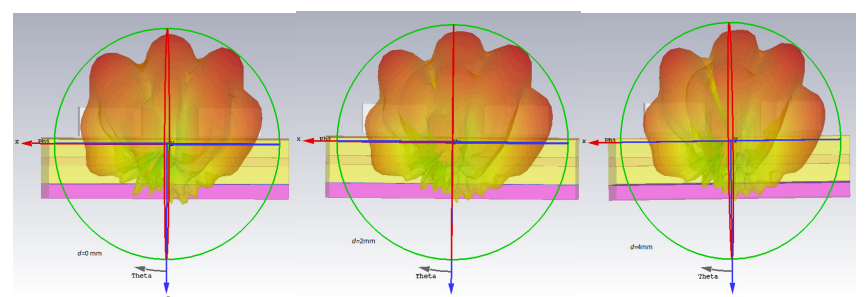




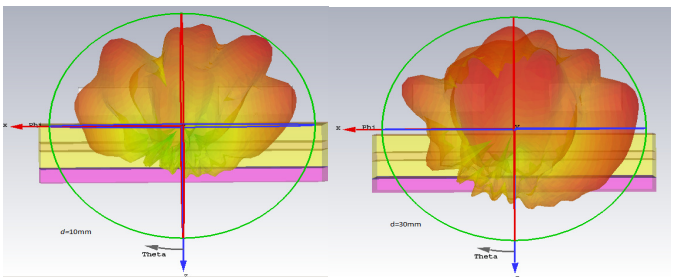

Figure 11. 3D Power pattern for different antenna-body distances as the antenna separation distance is $8 \mathrm{~cm}$.

When comparing channel responses and power patterns of different antenna location options, it is seen that the best small intestine coverage is achieved with $d=30 \mathrm{~mm}$ case as the $\mathrm{dB}$ range is set $0 \mathrm{~dB}--40 \mathrm{~dB}$. With $d=10 \mathrm{~mm}$, the channel responses are the strongest, but in this case small intestine coverage is minor. However, if we increase the $\mathrm{dB}$ range until $-50 \mathrm{~dB}$, the small intestine coverage is clearly wider also in $d=10 \mathrm{~mm}$ case. Furthermore, frequency has also an obvious impact on the propagation depth, as we can note by comparing power patterns of the case $d=10 \mathrm{~mm}$ at frequencies $f=4 \mathrm{GHz}, f=3.75 \mathrm{GHz}$, and $f=4.25 \mathrm{GHz}$ presented in Figure 12. In these cases, the $\mathrm{dB}$ range is $0--50 \mathrm{~dB}$. In this case, the small intestine coverage gets wider as the frequency increases

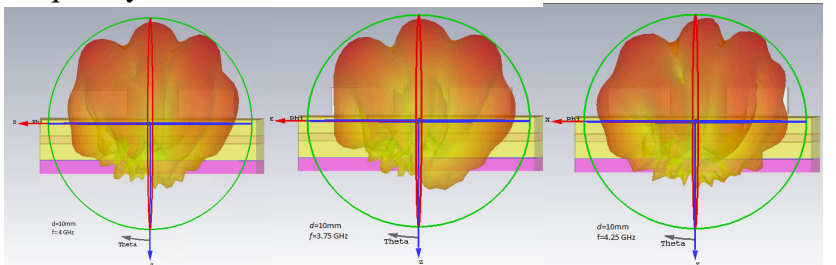

Figure 12. 3Dpower pattern for cases $d=10 \mathrm{~mm}$ and $d_{\mathrm{a}}=8 \mathrm{~cm}$ as the $\mathrm{dB}$ range is $0-50 \mathrm{~dB}$ with frequencies a) $f=4 \mathrm{GHz}, \mathrm{b}) f=3.75 \mathrm{GHz}$, and c) $f=4.25 \mathrm{GHz}$.

\section{SIMULATION RESULTS WITH VOXEL MODEL}

In this section, we present the simulation results with a female voxel model Laura in the antenna location option 1: side-by-side case. As seen from Figure 1b, antennas are slightly tilted to follow the skin surface, as it would be in the realistic case. Due to curved and pixelized form of the voxel model's abdomen area, there might be more space between the antenna and skin surface than in the layer model case, which can have influence on the channel strength. This issue is explained more in detail in [5].

S11 parameters, S21 response and impulse responses are presented in Figure $13 \mathrm{a}, \mathrm{b}$, and c, respectively. Interestingly, the variation between $\mathrm{S} 11 \mathrm{~s}$ for different antenna-body distances is minor than that with the layer model. There is also minor variation in the channel characteristics as well. One peculiarity is that at the antennas operational frequency $f=4 \mathrm{GHz}$, path loss is highest in the case of $d=30 \mathrm{~mm}$, whereas with the layer model the path loss was the second smallest. Path loss is smallest for the case $d=10 \mathrm{~mm}$, similarly to the layer model's case. Surprisingly small variation can be seen in the main peaks of the impulse responses. The main peak of the case $d=$ $30 \mathrm{~mm}$ is highest until $1.5 \mathrm{~ns}$, after which it decreases compared to the other cases. Interestingly, the largest variation between the different antenna-body distance cases can be found from time instant $2.5 \mathrm{~ns}$ onwards. The difference between the main peaks is even $25 \mathrm{~dB}$.

Next, we study 2D power flows with the voxel model in different antenna-body distance cases, presented for $\mathrm{dB}$ range $0--40 \mathrm{~dB}$ at $f=4 \mathrm{GHz}$ in Figure 14. The reason for choosing the $2 \mathrm{D}$ power presentation instead of $3 \mathrm{D}$ power presentation like in the layer model's case is that with 2D power plot we can observe power behavior in different selected crosscuts of the voxel model. Besides, arrows of the $2 \mathrm{D}$ power presentation are very informative when observing the propagation within the tissues.

From the power plots we can see, how well different parts of the small intestine area are reached with different antenna-body distances. For example, we take for our consideration the area marked as " $x$ " in the plots of Figure 14. As we can see, when the antenna-body distance is small, there is more arrows arriving to the point $\mathrm{X}$, whereas with $d=10 \mathrm{~mm}$ and $d=30 \mathrm{~mm}$ there is hardly any arrows around that area. This can further be validated by studying strength of the E-field at the point ' $x$ ', as shown in Figure 15. From the figures we can easily see that the strength of the E-field corresponds to the amount of values in the point ' $x$ ' at 4 GHz. The strength of the E-field is at lowest for $d=30 \mathrm{~mm}$ and $d=10 \mathrm{~mm}$, whereas strongest for $d=0 \mathrm{~mm}$. This result differs from those presented by the layer model, which is assumed to be due to different radiation due to tilt of the antennas and differences between the tissue shapes of the layer and voxel model. However, in some other point of the voxel model's small intestine, the result can be different. Due to lack of the space, other points are not considered in this paper and are left for the journal extension.

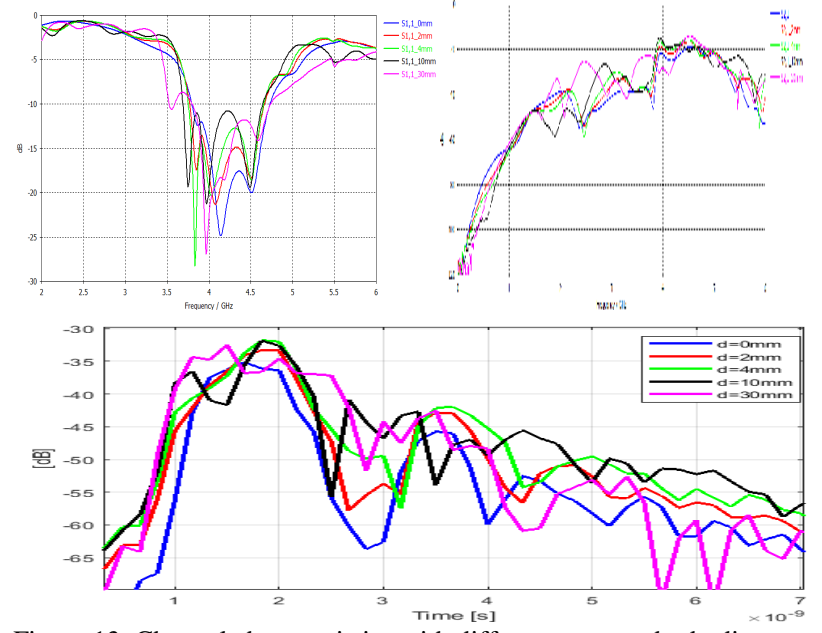

Figure 13. Channel characteristics with different antenna-body distances in a) frequency and b) time domain with the voxel model in the antenna location option 1 .

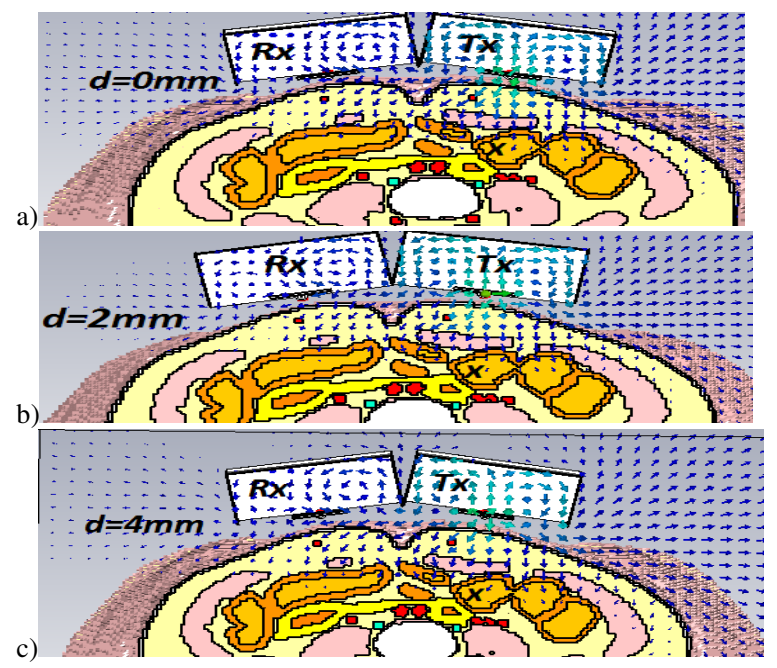




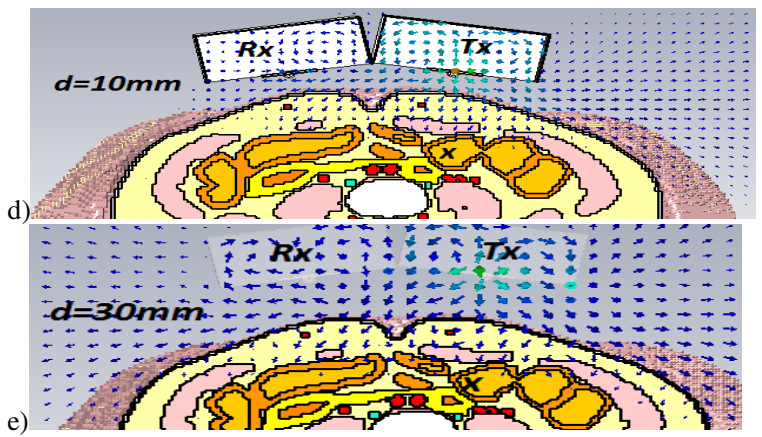

Figure 14a-e. 2D power flows for different antenna-body distances with the voxel model in the antenna location option 1.

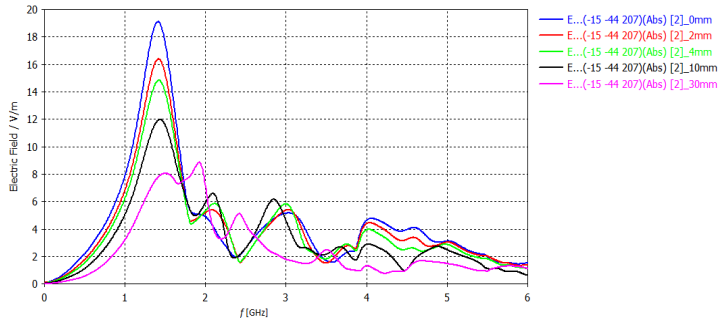

Figure 15. Strength of the field inside the small intestine point shown as black cross for different antenna-body distances.

\section{CONCLUSIONS}

This paper presented a simulation based study on the impact of the antenna-body distance on the on-body radio channel characteristics using highly directional antenna designed for inbody communications, e.g. capsule endoscopy localization. The on-body channel characteristics were evaluated both in frequency and time domains with several antenna-body distances as well as with several antenna location options. Furthermore, the impact of the antennabody distance was evaluated by studying power patterns, which also provide information about the propagation depth inside tissues in different antenna-body distances. It was shown that antenna-body distance has a significant impact on the on-body channel characteristics: in time domain the difference can be around $20 \mathrm{~dB}$ in the main peaks of the impulse responses and in frequency domain, the path loss difference can be even $30 \mathrm{~dB}$ within the frequency band of interest. Interestingly, the strongest channel between the Tx and $\mathrm{Rx}$ antennas is not always obtained in the cases of the largest skin-antenna distance, as it is usually expected. The antenna-body distance $d=1 \mathrm{~cm}$ seem to be optimal among these simulated cases. Furthermore, antenna-body distance has some impact on the propagation depth as well. The power patterns were studied within relatively strict $\mathrm{dB}$ range: only until $-40 \mathrm{~dB}$. With the layer model, the small intestine layer is achieved with all the antenna-body distance cases within the selected $\mathrm{dB}$ range. The best coverage of the small intestine area was obtained as the antenna-body distance was the largest. Instead, with the voxel model the result was vice versa: power flow hardly achieved the small intestine within the selected $\mathrm{dB}$ range as $d=10 \mathrm{~mm}$ and $d=30 \mathrm{~mm}$. The different results between the layer model and voxel model is assumed to be due to different antenna positions (antennas on the voxel model are tilted) and differences between the tissue shapes between the layer and voxel model. As we can see from the voxel model's power flow results, there are several propagation paths inside the human body.
For the realistic scenarios, the smaller antenna-body distance is always better, especially when considering the node antenna. The antenna attached on the monitoring device itself, the antenna-body distance could be slightly larger. Our future's work is to evaluate impact of antennabody distance with several different antennas designed for inbody communications as well as validate on-body layer model and voxel model simulation results with the measurements data.

\section{ACKNOWLEDGMENT}

This research has been financially supported by the project WBAN Communications in the Congested Environments and in part by Academy of Finland 6Genesis Flagship (grant 318927). Ilkka Virtanen, Timo Mäkinen, and Jari Sillanpää from University of Oulu deserve acknowledgement for their help to enable the exhaustive simulations. Furthermore, Dr. Markus Berg from CWC is acknowledged for his advices with the CST simulator.

\section{REFERENCES}

[1] P. Turalchuk, I. Munina, V. Pleskachev, V. Kirillov, O. Vendik, I. Vendik, "In-Body and On-Body Wave Propagation: Modeling and Measurements," International workshop on Antenna Technology: Small Antennas, Innovative Structures, and Applicattions (iWAT), 2017.

[2] P. A. Floor, R. Chavez-Santiago, S. Brovoll, O. Aardal, J. Bergsland, O-J. H. N. Grymyr, P. Halvorsen, R. Palomar, D. Plettemeier, S-E. Hamran, T. Ramstad, I. Balasingham, "In-Body to On-Body Ultrawideband Propagation Model Derived from Measurement in Living Animals," IEEE Journal of Biomedical and Health Informatics, 2015.

[3] S. Stoa, R. Chavez-Santiago, I. Balasinham, "An ultra wideband communication channel model for capsule endopscopy," ISABEL2014

[4] M. Särestöniemi, C. Kissi, C. Pomalaza Raez. M. Hämäläinen, J. linatti "Propagation and UWB channel characteristics on human abdomen area," accepted to be presented EUCAP2019.

[5] M. Särestöniemi; C. Kissi, C. Pomalaza-Raez, T. Kumpuniemi, M. Sonkki, S. Myllymäki, M. Hämäläinen, J. Iinatti; "Measurement and Simulation Based Study on the UWB Channel Characteristics on the Abdomen Area," submitted to ISMICT 2019 conference

[6] C. Kissi; M. Särestöniemi; C. Pomalaza-Raez; M. Sonkki; and M. N. Srifi, "Low-UWB directive antenna for Wireless Capsule Endoscopy localization", BodyNets2018, Oulu, Finland, October 2018.

[7] C. Kissi; M. Särestöniemi; T. Kumpuniemi, M. Sonkki, S. Myllymäki, M. N. Scrifi, C. Pomalaza-Raez; "Low-UWB antennas in the Vicinity of Human Body," submitted to ISMICT 2019 conference.

[8] N. Siauve, R. Scorretti,N. Burais, L. Nicolas, A. Nicolas, "Electroimagnetic Fields and Human Body:a New Challenge for Electromagnetic Computation,'International journal for computation and mathematics in electrical and electronic engineering, 2003.

[9] T. Tuovinen, T. Kumpuniemi, K. Y .Yazdandoost, M. Hämäläinen, J. Iinatti, "Effect of the Antenna-Human Body Distance on the Antenna Matching in UWB WBAN Applications,'ISMICT2013.

[10] David Ferreira, Pedro Pires, Ruben Rodrigues, Rafael F.S. Caldeirinha, "Wearable Textile Antennas: Examining the effect of bending on their performance.", Antennas and Propagation Magazine IEEE, vol. 59, no. 3, pp. 54-59, 2017.

[11] N. A. Elias, N. A. Samsuri, M. K. A. Rahim, N. Othman, "The Effects of Human Body and Bending on Dipole Textile Antenna Performance and SAR", Proceeding of APMC 2012, 2012.

[12] T. B. Welch, R. L. Musselman, B. Emissiene, P. Gift, D. Choudhury, D. N. Cassadine, S. Yano, "The Effects of the Human Body on UWB Signal Propagation in an Indoor Environment," IEEE Journal on Selected Areas in Communication, Dec. 2002.

[13] CST Microwave studio, www. cst.com.

[14] https://www.itis.ethz.ch/virtual-population/tissueproperties/database/dielectric-properties/ 